\title{
Cosmic Evolution in Self-Interacting Brans-Dicke Cosmology
}

\author{
Muhammad SHARIF *and Saira WAHEED ${ }^{\dagger}$ \\ Department of Mathematics, University of the Punjab, \\ Quaid-e-Azam Campus, Lahore-54590, Pakistan.
}

\begin{abstract}
In this paper, we investigate the nature of self-interacting potential that can support the accelerated expansion of the universe as indicated by observational data. For this purpose, we consider the Bianchi type I (BI) universe model in the Brans-Dicke (BD) field scenario and use a power law for both the scalar field and the scale factor. It is found that the accelerated expansion of the universe can be discussed for a positive power law potential with negative values of the BD parameter. We also explore the evolution of energy density perturbations. This analysis indicates that the model allows growing modes for negative values of the $\mathrm{BD}$ parameter and $m>0$.
\end{abstract}

Keywords: Brans-Dicke theory, scalar field, cosmic evolution, PACs numbers: 04.50.Kd; 98.80.-k

\section{Introduction}

Many astronomical experiments such as Supernova (Ia) ${ }^{1,2)}$, Wilkinson Microwave Anisotropy Probe (WMAP) ${ }^{3)}$, Sloan Digital Sky Survey (SDSS) ${ }^{4}$, galactic cluster emission of X-rays ${ }^{5}$ ), large scale-structure ${ }^{6)}$ and weak lensing 7), provide evidence of an accelerated expansion of the universe in the present

*Email address: msharif.math@pu.edu.pk

†smathematics@hotmail.com 
phase. As the baryonic matter and dark matter do not provide the total energy density of the universe, it has been suggested that there is some kind of "missing energy". The Chaplygin gas, phantom, quintessence and cosmological constant ${ }^{8,9}$ ) are some appropriate candidates of dark energy (missing energy), considered to be responsible for the expansion of the universe. In order to conciliate the nucleosynthesis scenario in the radiation-dominated era, it is argued that the universe was expanding with deceleration in its early phase.

Quintessence is a dynamical slowly evolving spatially inhomogeneous component of energy density with a negative pressure ${ }^{9)}$ having an EoS parameter lying between 0 and -1 . However, this model was proved to be not very successful as it faces problems like fine tuning and cosmic coincidence ${ }^{10)}$. To solve these problems, a special form of quintessence field called the tracker field (with a wide range of initial values of $\rho_{\phi}$, such fields roll down along a common evolutionary track with the present energy density of the universe $\rho_{n}$ and end up in the observable universe with $\rho_{\phi}$ comparable to $\rho_{n}$ in the present epoch) has been constructed ${ }^{11)}$. Different types of potential have been developed ${ }^{12}$ ) to understand the nature of dark energy using a quintessence model but each of them has its own drawbacks.

Scalar-tensor theories of gravity as modified gravity theories are proved to be very effective in explaining many outstanding problems in cosmology 13-16) like inflation, the "early- and late-" time behaviors of the universe, coincidence problem and cosmic acceleration. Brans and Dicke ${ }^{17)}$ proposed a scalar-tensor generalization of general relativity (GR) by introducing a timevarying gravitational constant (as $G(t)=\frac{1}{\phi(t)}$ ) and the direct interaction of a scalar field with a geometry known as the BD theory of gravity. The weak equivalence principle, Mach's principle ${ }^{18)}$ and Dirac's large number hypothesis ${ }^{19)}$ are also accommodated in this theory. Using local gravity tests, it has been shown ${ }^{20,21)}$ that the generic dimensionless BD parameter $\omega$ should be very large, i.e., $\omega \geq 40,000$, for being compatible with solar system experiment bounds. This theory goes over to GR if $\omega \rightarrow \infty$ and the scalar field is constant ${ }^{22}$ ). In general, it has been shown ${ }^{23,24)}$ that the BD theory is reducible to GR only if the trace of the energy-momentum tensor $T^{(m)}$ does not vanish, i.e., $T^{(m)} \neq 0$.

Many researchers have explored the cosmic evolution of the universe in the BD theory. In the GR and scalar tensor theories, much work has been done using Bianchi models to discuss different stages of the cosmic evolution ${ }^{25,26)}$. In ref. 27 , it has been pointed out that some large-angle anomalies are 
seen in CMB radiations that violate the statistical isotropy of the observable universe. For a better description of these anomalies, plane symmetric and homogeneous but anisotropic universe models play a very significant role. Moreover, it is pointed out ${ }^{28)}$ that removing a Bianchi component from WMAP data can explain various large-angle anomalies yielding an isotropic universe. Thus, the universe may have accomplished a slight anisotropic geometry in cosmological models irrespective of inflation. King and Coles 29) described the evolution of magnetized axisymmetric Bianchi I universe in the presence of vacuum energy. Singh and Rai ${ }^{30)}$ constructed various BD cosmological models using Bianchi models with a perfect fluid to explain the evolution of the universe. In our recent study ${ }^{31)}$, we have investigated some Bianchi type I universe models using perfect, anisotropic and magnetized anisotropic fluids in self-interacting BD cosmology. Bermann ${ }^{32)}$ investigated different cosmological models using a constant deceleration parameter. Khoury and Weltman ${ }^{33)}$ explored the coupling of a scalar field with matter of unity order, which is allowed to evolve cosmologically.

Bertolami and Martins ${ }^{13)}$ obtained accelerated expanding solutions using quadratic potentials. They also evaluated energy density perturbations and discussed the growing modes of their model. By extending the concept of quintessence to flat non minimally coupled scalar tensor theories, Ritis et al. ${ }^{34)}$ found a family of exact solutions parameterized by the parameter 's'. These solutions are proposed as a class of tracker fields known as tracking solutions. Sen and Sen ${ }^{35)}$ investigated accelerating solutions using a potential with a time-dependent mass squared term in $\mathrm{BD}$ cosmology. By introducing the non minimal coupling term $\psi R \frac{\phi^{2}}{2}$, different kinds of potential have been discussed ${ }^{36)}$. Sen and Seshadri ${ }^{37)}$ investigated the nature of self-interacting potential as well as the evolution of energy density perturbations in this theory.

In this study, we explore the nature of self-interacting potential and $\mathrm{BD}$ field energy density as dark energy, which can account for the accelerated expansion of the universe by taking a perfect fluid and the LRS Bianchi type I (BI) universe into account. This paper is organized as follows. In the next section, we formulate the field equations of the self-interacting BD theory for the BI universe. We discuss solutions by taking a power law ansatz. In $\S 3$, we provide the evolution of energy density perturbations by taking a perturbed form of field equations. We discuss the results in the last section. 


\section{Self-Interacting Potential and Dark Energy}

The action for the BD theory with the self-interacting potential $V(\phi)$ is given by ${ }^{37)}$

$$
S=\int d^{4} x \sqrt{-g}\left[\phi R-\frac{\omega_{0}}{\phi} \phi^{, \alpha} \phi_{, \alpha}-V(\phi)+L_{m}\right], \quad \alpha=0,1,2,3 .
$$

Here, $L_{m}$ is the matter part of the Lagrangian and $\omega_{0}$ is the constant BD coupling parameter (where the units $8 \pi G_{0}=c=1$ are imposed). The corresponding field equations derived from the action (11) are

$$
\begin{aligned}
G_{\mu \nu} & =\frac{\omega_{0}}{\phi^{2}}\left[\phi_{, \mu} \phi_{, \nu}-\frac{1}{2} g_{\mu \nu} \phi_{, \alpha} \phi^{, \alpha}\right]+\frac{1}{\phi}\left[\phi_{, \mu ; \nu}-g_{\mu \nu} \square \phi\right]+\frac{T_{\mu \nu}}{\phi}+\frac{V(\phi)}{2 \phi}, \\
\square \phi & =\frac{T}{3+2 \omega_{0}}-\frac{2}{3+2 \omega_{0}}\left[V(\phi)-\phi \frac{V(\phi)_{, \phi}}{2}\right] .
\end{aligned}
$$

The terms $T=g^{\mu \nu} T_{\mu \nu}, \square=\Delta^{\mu} \Delta_{\mu}$ and $\Delta^{\mu}$ represent the trace of the energymomentum tensor, the d'Alembertian operator, and the covariant derivative respectively.

The homogeneous, anisotropic, and spatially flat BI universe model is given by ${ }^{38)}$

$$
d s^{2}=d t^{2}-A^{2}(t) d x^{2}-B^{2}(t)\left(d y^{2}+d z^{2}\right),
$$

where $A$ and $B$ are the scale factors along the transverse direction $x$ and the two equivalent longitudinal directions $y$ and $z$, respectively. The energymomentum tensor for a perfect fluid is

$$
T_{\mu \nu}=(\rho+P) u_{\mu} u_{\nu}-P g_{\mu \nu}
$$

where $\rho$ and $P$ are the energy density and pressure, respectively, and $u^{\mu}$ is the four-velocity with the normalization condition $u_{\mu} u^{\nu}=1$. The energy conservation equation is

$$
\dot{\rho}+\left(\frac{\dot{A}}{A}+2 \frac{\dot{B}}{B}\right)(\rho+P)=0 .
$$


The corresponding BD field equations become

$$
\begin{aligned}
& \frac{2 \dot{A} \dot{B}}{A B}+\frac{\dot{B}^{2}}{B^{2}}=\frac{\rho}{\phi}+\frac{\omega_{0}}{2} \frac{\dot{\phi}^{2}}{\phi^{2}}-\left(\frac{\dot{A}}{A}+2 \frac{\dot{B}}{B}\right) \frac{\dot{\phi}}{\phi}+\frac{V(\phi)}{2 \phi}, \\
& 2 \frac{\ddot{B}}{B}+\frac{\dot{B}^{2}}{B^{2}}=-\frac{P}{\phi}-\frac{\omega_{0}}{2} \frac{\dot{\phi}^{2}}{\phi^{2}}-2 \frac{\dot{B}}{B} \frac{\dot{\phi}}{\phi}-\frac{\ddot{\phi}}{\phi}+\frac{V(\phi)}{2 \phi}, \\
& \frac{\ddot{B}}{B}+\frac{\ddot{A}}{A}+\frac{\dot{A} \dot{B}}{A B}=-\frac{P}{\phi}-\frac{\omega_{0}}{2} \frac{\dot{\phi}^{2}}{\phi^{2}}-\frac{\ddot{\phi}}{\phi}-\left(\frac{\dot{A}}{A}+\frac{\dot{B}}{B}\right) \frac{\dot{\phi}}{\phi}+\frac{V(\phi)}{2 \phi},
\end{aligned}
$$

while the Klein-Gordan equation for scalar field is

$$
\ddot{\phi}+\left(\frac{\dot{A}}{A}+2 \frac{\dot{B}}{B}\right) \dot{\phi}=\frac{\rho-3 P}{2 \omega_{0}+3}-\frac{2}{2 \omega_{0}+3}\left(V-\frac{\phi V_{, \phi}}{2}\right) .
$$

The average scale factor and mean Hubble parameter respectively turn out to be

$$
a^{3}(t)=A B^{2}, \quad H(t)=\frac{1}{3}\left(\frac{\dot{A}}{A}+2 \frac{\dot{B}}{B}\right),
$$

whereas the expansion and shear scalar are

$$
\theta=u_{; a}^{a}=\frac{\dot{A}}{A}+2 \frac{\dot{B}}{B}, \quad \sigma=\frac{1}{\sqrt{3}}\left(\frac{\dot{A}}{A}-\frac{\dot{B}}{B}\right) .
$$

It is reported in ref. 39 that, for a spatially homogeneous universe model, the normal congruence to homogeneous expansion yields the ratio $\frac{\sigma}{\theta}$ as a constant, i.e., "the expansion scalar $\theta$ is proportional to the shear scalar $\sigma$ ". This physical condition leads to the following relation between the scale factors:

$$
A=B^{m}
$$

where $m \neq 1$ is any constant (for $m=1$, it reduces to a flat FRW model). This condition has been used to investigate various exact universe models 
40-42). This assumption leads to the following set of BD dynamical equations:

$$
\begin{aligned}
(2 m+1) \frac{\dot{B}^{2}}{B^{2}} & =\frac{\rho}{\phi}+\frac{\omega_{0}}{2} \frac{\dot{\phi}^{2}}{\phi^{2}}-(m+2) \frac{\dot{B}}{B} \frac{\dot{\phi}}{\phi}+\frac{V(\phi)}{2 \phi} \\
2 \frac{\ddot{B}}{B}+\frac{\dot{B}^{2}}{B^{2}} & =-\frac{P}{\phi}-\frac{\omega_{0}}{2} \frac{\dot{\phi}^{2}}{\phi^{2}}-2 \frac{\dot{B}}{B} \frac{\dot{\phi}}{\phi}-\frac{\ddot{\phi}}{\phi}+\frac{V(\phi)}{2 \phi} \\
(m+1) \frac{\ddot{B}}{B}+m^{2} \frac{\dot{B}^{2}}{B^{2}} & =-\frac{P}{\phi}-\frac{\omega_{0}}{2} \frac{\dot{\phi}^{2}}{\phi^{2}}-\frac{\ddot{\phi}}{\phi}-(m+1) \frac{\dot{B}}{B} \frac{\dot{\phi}}{\phi}+\frac{V(\phi)}{2 \phi} \\
\ddot{\phi}+(m+2) \frac{\dot{B}}{B} \dot{\phi} & =\frac{\rho-3 P}{2 \omega_{0}+3}-\frac{2}{2 \omega_{0}+3}\left(V-\frac{\phi V_{, \phi}}{2}\right) \\
\dot{\rho} & =-(m+2) \frac{\dot{B}}{B}(\rho+P) .
\end{aligned}
$$

Here, the unknown functions are $B, V, \rho, P$ and $\phi$, and there are only three independent field equations. In order to have a closed system of equations, we take the power laws for the scale factor and scalar field as

$$
B=b_{0}\left(\frac{t}{t_{0}}\right)^{\alpha}, \quad \phi=\phi_{0}\left(\frac{t}{t_{0}}\right)^{\beta},
$$

where $t_{0}, b_{0}$, and $\phi_{0}$ indicate the present time, scale factor and scalar field, respectively. The deceleration parameter is given by

$$
q=-\left(1-\frac{3}{\alpha(m+2)}\right)
$$

For an accelerated expanding solution of the field equations, the parameter $\alpha$ must be greater than $\frac{m+2}{3}$. Adding eqs.(12) and (13), it follows that

$$
(m+3) \frac{\ddot{B}}{B}+\left(m^{2}+1\right) \frac{\dot{B}^{2}}{B^{2}}=-\omega_{0}\left(\frac{\dot{\phi}}{\phi}\right)^{2}-(m+3) \frac{\dot{B}}{B} \frac{\dot{\phi}}{\phi}-2 \frac{\ddot{\phi}}{\phi}-2 \frac{P}{\phi}+\frac{V(\phi)}{\phi} .
$$

Equation (15) leads to

$$
\rho+P=-\frac{\dot{\rho} B}{(m+2) \dot{B}} .
$$

Subtracting eq.(11) from eq.(17) and substituting the power law ansatz given by eq.(16) along with eq.(18) in the resulting equation, the energy density for matter can be written as

$$
\rho=\rho_{c(m)} t^{\beta-2}
$$


where

$$
\begin{aligned}
\rho_{c(m)} & =\frac{(m+2) \phi_{0} \alpha}{(\beta-2) t_{0}^{\beta}}\left[\frac{\left(m^{2}-3 m+2\right) \alpha^{2}}{2}-\frac{\beta(m \alpha+\alpha+2)}{2}-\frac{(m+3) \alpha}{2}\right. \\
& \left.+\left(1+\omega_{0}\right) \beta^{2}\right] .
\end{aligned}
$$

Using the energy density given by eq.(19) in eq.(15), the corresponding pressure becomes

$$
P=P_{c(m)} t^{\beta-2}
$$

where

$$
\begin{aligned}
P_{c(m)} & =\frac{\phi_{0}(2-\beta-(m+2) \alpha)}{(\beta-2) t_{0}^{\beta}}\left[\frac{\left(m^{2}-3 m+2\right) \alpha^{2}}{2}-\frac{\beta(m \alpha+\alpha+2)}{2}\right. \\
& \left.-\frac{(m+3) \alpha}{2}+\left(1+\omega_{0}\right) \beta^{2}\right] .
\end{aligned}
$$

From these expressions, we can write EoS as

$$
P=\gamma_{B} \rho, \quad \gamma_{B}=\frac{2-\beta}{\alpha(m+2)}-1,
$$

where $\gamma_{B}$ is the EoS parameter. Since the fluid under consideration is a perfect fluid $\left(0<\gamma_{B}<1\right)$, it constrains the parameter $\beta$ as

$$
2-2 \alpha(m+2)<\beta<2-\alpha(m+2) .
$$

We would like to mention here that for any positive $m$ other than 1 and $\alpha>\frac{3}{m+2}, \beta$ remains negative.

Solving the field equations (11)-(14) for the self-interacting potential, we obtain

$$
V(\phi)=V_{c} \phi^{\frac{(\beta-2)}{\beta}},
$$

where

$$
\begin{aligned}
V_{c} & =\frac{\phi_{0}^{2 / \beta}}{t_{0}^{2}}\left[\alpha^{2}\left\{\frac{m^{2}+5 m+6}{2}-\left(\frac{2 \alpha(m+2)-2+\beta}{2-\beta}\right)\left(\frac{m^{2}-3 m+2}{2}\right)\right\}\right. \\
& +\alpha\left(\frac{m+3}{2}\right) \frac{(2 \beta+2 \alpha(m+2)-4)}{2-\beta}+\beta\left\{\frac{(3 m+7) \alpha}{2}-1+\frac{(m+1) \alpha+2}{2}\right. \\
& \left.\left.\times \frac{2 \alpha(m+2)-2+\beta}{(2-\beta)}\right\}+\beta^{2}\left(1-\frac{\left(1+\omega_{0}\right)(2 \alpha(m+2)-2+\beta)}{(2-\beta)}\right)\right] .
\end{aligned}
$$


Now, we discuss the energy density and pressure for the missing energy (dark energy) by taking the energy density and pressure due to the scalar field. These are given by

$$
\begin{aligned}
\rho_{\phi} & =\frac{\omega_{0}}{2} \frac{\dot{\phi}^{2}}{\phi}+\frac{V}{2}-\frac{(m+2) \dot{B} \dot{\phi}}{B} \\
P_{\phi} & =\frac{\omega_{0}}{2} \frac{\dot{\phi}^{2}}{\phi}-\frac{V}{2}+\ddot{\phi}+\frac{(m+3) \dot{B} \dot{\phi}}{2 B} .
\end{aligned}
$$

After inserting their respective values, these quantities become

$$
\rho_{\phi}=\rho_{c(\phi)} t^{\beta-2}, \quad P_{\phi}=P_{c(\phi)} t^{\beta-2},
$$

where

$$
\begin{aligned}
\rho_{c(\phi)} & =\left[\frac{\omega_{0} \beta^{2}}{2}-\alpha \beta(m+2)+\frac{1}{2}\left(\alpha ^ { 2 } \left\{\frac{m^{2}+5 m+6}{2}-\left(\frac{m^{2}-3 m+2}{2}\right)\right.\right.\right. \\
& \left.\times\left(\frac{2 \alpha(m+2)-2+\beta}{2-\beta}\right)\right\}+\alpha \frac{(m+3)}{2} \frac{(2 \beta+2 \alpha(m+2)-4)}{2-\beta} \\
& +\beta\left\{\frac{(3 m+7) \alpha}{2}-1+\frac{((m+1) \alpha+2)}{2} \frac{(2 \alpha(m+2)-2+\beta)}{(2-\beta)}\right\} \\
& \left.+\beta^{2}\left(1-\frac{\left(1+\omega_{0}\right)(2 \alpha(m+2)-2+\beta)}{(2-\beta)}\right)\right] \frac{\phi_{0}}{t_{0}^{\beta}}, \\
P_{c(\phi)} & {\left[\frac{\omega_{0} \beta^{2}}{2}+\alpha \beta \frac{(m+3)}{2}+\beta^{2}-\beta-\frac{1}{2}\left(\alpha ^ { 2 } \left\{\frac{m^{2}+5 m+6}{2}-\left(\frac{m^{2}-3 m}{2}\right.\right.\right.\right.} \\
+ & \left.1)\left(\frac{2 \alpha(m+2)-2+\beta}{2-\beta}\right)\right\}+\alpha \frac{(m+3)}{2} \frac{(2 \beta+2 \alpha(m+2)-4)}{2-\beta} \\
+ & \beta\left\{\frac{(3 m+7) \alpha}{2}-1+\frac{((m+1) \alpha+2)}{2} \frac{(2 \alpha(m+2)-2+\beta)}{(2-\beta)}\right\} \\
+ & \left.\beta^{2}\left(1-\frac{\left(1+\omega_{0}\right)(2 \alpha(m+2)-2+\beta)}{(2-\beta)}\right)\right] \frac{\phi_{0}}{t_{0}^{\beta}} .
\end{aligned}
$$

The energy density and pressure for the scalar field obey the EoS, i.e., $P_{\phi}=$ $\gamma_{\phi} \rho_{\phi}$, where

$$
\gamma_{\phi}=-1+\frac{\beta^{2}\left(1+\omega_{0}\right)-\beta-\frac{(m+1)}{2} \alpha \beta}{\rho_{c}} .
$$


In order to have a positive energy density for both matter and scalar field, the BD parameter should be constrained as

$$
\begin{aligned}
& \frac{2(2-\beta)}{\beta^{2}(4-2 \beta-2 \alpha(m+2))}\left[\alpha \beta(m+2)-\frac{\left(m^{2}+5 m+6\right) \alpha^{2}}{4}+\left(\frac{m^{2}-3 m}{4}\right.\right. \\
& \left.\times \alpha^{2}+\frac{\alpha^{2}}{2}\right) \frac{(2 \alpha(m+2)+\beta-2)}{2-\beta}-\frac{\alpha(m+3)(2 \alpha(m+2)+2 \beta-4)}{4(2-\beta)} \\
& -\frac{\beta(3 m+7) \alpha}{4}+\frac{\beta}{2}-\frac{\beta(2 \alpha(m+2)+\beta-2)(2+\alpha(m+1))}{4(2-\beta)}-\frac{\beta^{2}}{2} \\
& \left.+\frac{\beta^{2}(2 \alpha(m+2)-2+\beta)}{2(2-\beta)}\right]<\omega_{0}<\left[\frac{2+\alpha(m+1)}{2 \beta}+\frac{\alpha(m+3)}{2 \beta^{2}}-1\right. \\
& \left.-\frac{\alpha^{2}\left(m^{2}-3 m+2\right)}{2 \beta^{2}}\right] .
\end{aligned}
$$

The deceleration parameter requires $\alpha>\frac{m+2}{3}$ for an accelerated expansion of the universe. Since $m>0,(m \neq 1)$, we can discuss two possible values of $m$, i.e., $m>1$ and $0<m<1$. The feasible region for $\omega_{0}$ allowed by eq.(30) as $\left(\beta, \omega_{0}\right)$ space is shown in Fig. 1. Fig. 1(a) indicates the $\left(\beta, \omega_{0}\right)$ space for $m>1$ and Fig. 1(b) shows the allowed region for these parameters with $0<m<1$. These figures indicate that the positivity condition for both energy densities implies negative values of the BD parameter in both cases. We found that the admissible range of $\mathrm{BD}$ parameter turns out to be $-1.5<\omega_{0}<-1$ only for $0<m<1$ with $\beta<-5.5$. This range for the BD parameter is negative. However, it does not yield ghost instabilities. A ghost cannot exist consistently, however, in the present model, the ghost field could be avoided by a suitable selection of parameters.

Using eq.(11), the matter and scalar field density parameters are defined as

$$
\Omega_{m}=\frac{\rho_{m} B^{2}}{\phi(1+2 m) \dot{B}^{2}}, \quad \Omega_{\phi}=\frac{\rho_{\phi} B^{2}}{\phi(1+2 m) \dot{B}^{2}} .
$$

Inserting $B$ and $\phi$ from eq.(16), it follows that

$$
\Omega_{m}=\frac{\rho_{c(m)}}{(1+2 m) \alpha^{2}}, \quad \Omega_{\phi}=\frac{\rho_{c(\phi)}}{(1+2 m) \alpha^{2}} .
$$

In terms of matter and the scalar density parameters $\Omega_{m}$ and $\Omega_{\phi}$, the EoS parameter $\gamma_{\phi}$ in eq.(29) can be rewritten as

$$
\gamma_{\phi}=-1-\frac{\frac{\alpha \beta(1+m)}{2}-\frac{(\beta-2) t_{0}^{\beta} \alpha(1+2 m) \Omega_{m}}{(m+2) \phi_{0}}+\frac{\left(m^{2}-3 m+2\right) \alpha^{2}}{2}-\frac{(m+3) \alpha}{2}}{(1+2 m) \alpha^{2} \Omega_{\phi}} .
$$



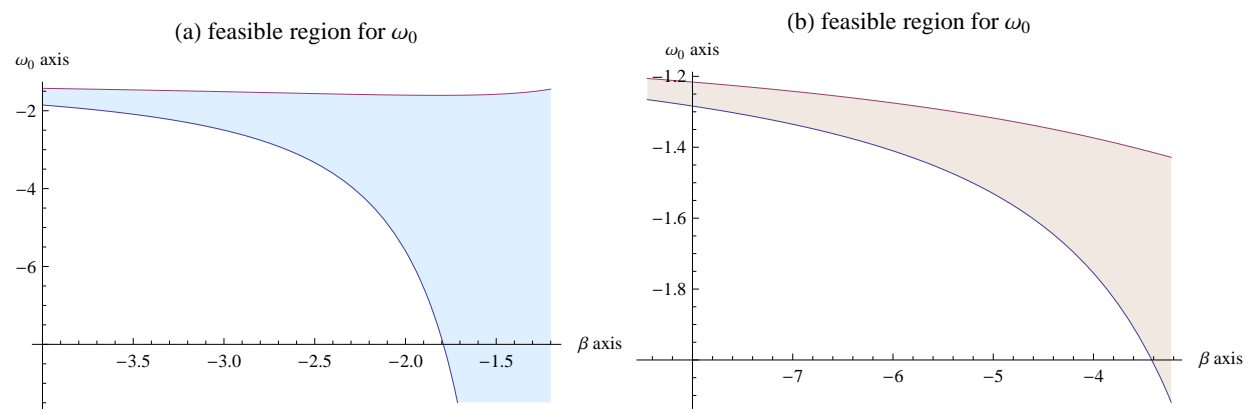

Figure 1: Admissible region for $\omega_{0}$ vs $\beta$ for (a) $m=2$ and $\alpha=0.8$, (b) $m=3 / 4$ and $\alpha=1.1$ (Color online).
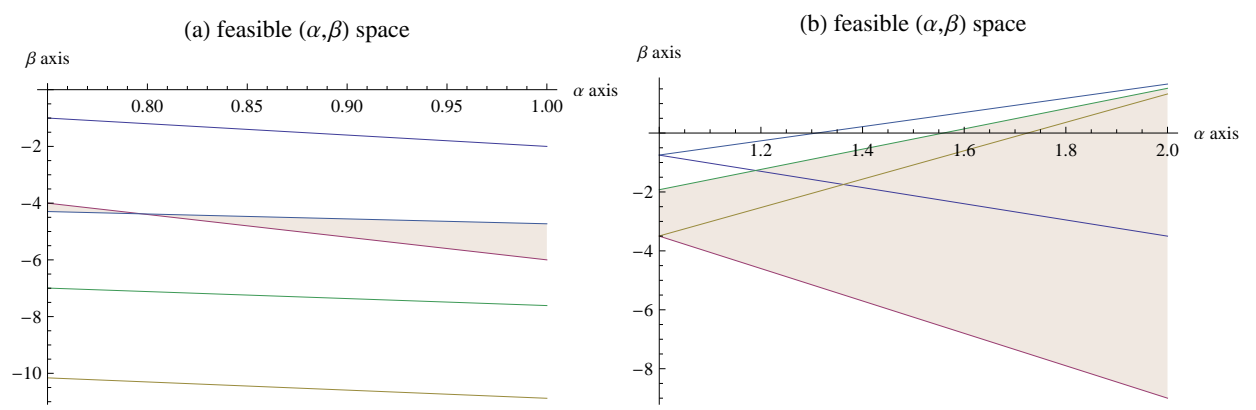

Figure 2: Allowed region for parameter $\beta$ versus $\alpha$ with (a) $\gamma_{\phi}=-1.24, m=$ 2 and (b) $\gamma_{\phi}=-0.6, m=3 / 4$ (Color online).

The observational data provided by the Supernova Cosmology Project and High $z$ Survey Project can lead to an accelerated expanding universe with a positive cosmological constant ${ }^{1,2)}$. By taking the best-fit values of the density parameters for matter and the scalar field (for flatness of the universe, i.e., $\left.\Omega_{m}=0.28, \Omega_{\phi}=0.72\right)^{37)}$ as well as the bounds for $\beta$ given by eq.(23), we can determine the range for the EoS parameter $\gamma_{\phi}$. We consider the case when $0<m<1$ and take $m=3 / 4$. The corresponding constraints for the other parameters are $\alpha>1.09$ and $-8.45<\beta<-3.225$. Using these values, the constraint for the EoS parameter $\gamma_{\phi}$ turns out to be $-1.44<$ $\gamma_{\phi}<0.71$. Likewise, in the case of $m>1$, we take $m=2$ and thus we have $\alpha>0.75$ and $-4.4<\beta<-1.2$. The corresponding bounds for $\gamma_{\phi}$ will be $-1.8<\gamma_{\phi}<-0.23$. The allowed region in the $(\alpha, \beta)$ space with $0.2<\Omega_{m}<0.4$, which satisfies $-1.44<\gamma_{\phi}<0.71$ and $-1.8<\gamma_{\phi}<-0.23$, 

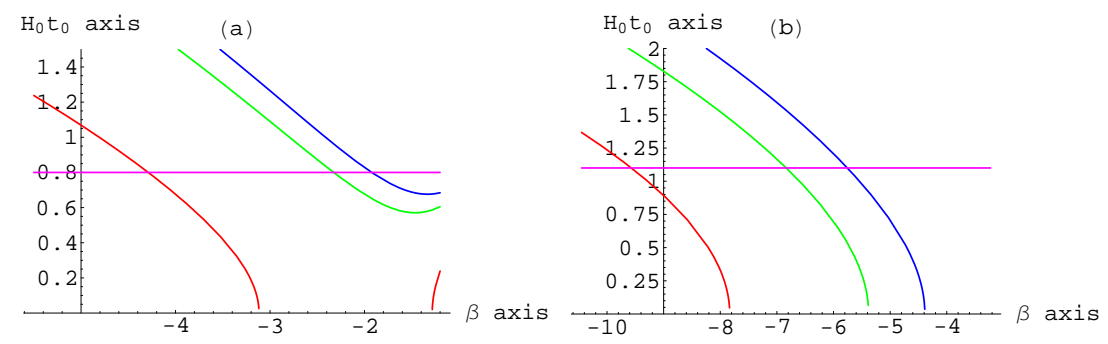

Figure 3: (a) Plot of $H_{0} t_{0}$ vs $\beta$ for $\alpha=0.8, \Omega_{m}=0.28$, and $m=2$. Here, the pink horizontal line corresponds to $\alpha=0.8$. (b) Plot of $H_{0} t_{0}$ vs $\beta$ for $\alpha=1.1, \Omega_{m}=0.28$, and $m=3 / 4$. Here, the pink horizontal line indicates $\alpha=1.1$ (Color online).

is shown as a shaded region in Fig. 2. In Fig. 2(a), we take the case $m>1$, and Fig. 2(b) corresponds to the case $0<m<1$.

As the BD theory of gravity has a salient feature of a time-varying gravitational constant, i.e., $G(t)=\frac{1}{\phi(t)}$, the rate of change in the gravitational constant at the present time can be calculated as

$$
\left[\frac{\dot{G}}{G}\right]_{t=t_{0}}=-\frac{\beta}{\alpha} H_{0}
$$

where $H_{0}$ is the directional Hubble parameter $\frac{\dot{B}}{B}$ at the present time and we have used the power law ansatz eq.(16). For a viable model representing the accelerated expansion of the universe, the constraint $\left[\frac{\dot{G}}{G}\right]_{t=t_{0}}<10^{-10}$ should be satisfied ${ }^{39)}$. For our model, the best-fit value of the parameter $\alpha=H_{0} t_{0}$ admitting the accelerated expansion can be written as

$$
\begin{aligned}
\left(H_{0} t_{0}\right)^{2} & =\frac{\alpha(m+2)}{(1+2 m) \Omega_{m}}\left[\frac{m^{2}-3 m+2}{2} \alpha^{2}-\frac{\beta(m \alpha+\alpha+2)}{2}-\frac{(m+3) \alpha}{2}\right. \\
& \left.+\left(1+\omega_{0}\right) \beta^{2}\right] .
\end{aligned}
$$

The graphical behavior of this expression in the cases $0<m<1$ and $m>1$ along with appropriate values of other parameters is shown in Fig. 3. It can be seen that the graphs of $H_{0} t_{0}$ with the allowed range of $\omega_{0}$ intersect at least at one point on the respective horizontal lines $\alpha=0.8$ and $\alpha=1.1$ in both cases.

The correction to Newton's law leads to two kind of problems: the variation in the gravitational constant and the propagation of the fifth force. From 
the cosmological point of view, the gravitational constant should be positive (it restricts $\phi_{0}$ to be positive) and its variation should be constrained within certain limits ${ }^{13-15)}$. The case of a constant BD parameter yields small variations that should be constrained by $\left[\frac{\dot{G}}{G}\right]_{0}<4 \times 10^{-10}$ yrs. In our case, the expression is given by $\left[\frac{\dot{G}}{G}\right]_{0}=-\frac{\beta}{t_{0}}$, where the present time is taken to be $t_{0}=14 \pm 2$ Gyr. By choosing the appropriate values of the parameters, it can be shown that in our case this constraint can be easily satisfied. For example, if we take $\alpha=0.8, m=2$ and $\beta=-1.3$ as $-4.4<\beta<-1.2$, then we have $\left[\frac{\dot{G}}{G}\right]_{0}=0.93 \times 10^{-10}$, which safely lies within the limit $<4 \times 10^{-10}$. Thus it can be concluded that, in our case, the variation in the gravitational constant (due to presence of scalar field) could satisfy the observational limit suggested for cosmic acceleration.

The Brans-Dicke theory in the Jordan frame can be transformed to the Einstein frame by defining a conformal transformation. The resulting action in the Einstein frame corresponds to a coupled quintessence field scenario with the coupling given by $2 Q^{2}=\frac{1}{2 \omega_{B D}+3}$ (this leads to a constant coupling $Q=-\frac{1}{\sqrt{6}}$ in metric $f(R)$ gravity) ${ }^{43-47)}$. It is argued that when this coupling is of order unity, local gravity tests become inconsistent owing to the propagation of fifth force between the field and the non relativistic matter. Whenever the gravity is modified from GR, there are some constraints that come from the local gravity tests like solar system tests, and the violation of the equivalence principle etc.

The action of the BD theory in the Jordan frame can be transformed to the Einstein frame via conformal transformation defined by the coupling factor $F=e^{-2 Q \phi}$ (basically, we define $g_{\mu \nu}=F(\phi) g_{\mu \nu}$ ). For a massless scalar field, the coupling factor should be constrained by the condition $|Q|<2.5 \times$ $10^{-3}$ that comes from the experimental bound on the BD parameter $\omega_{B D}>$ 40, 000. When the field is massive, this coupling factor can be large $|Q| \sim 1$, consequently, the Chameleon mechanism provides a way to be consistent with local gravity tests. In this regard, the field mass needs to be small in order to realize the present acceleration on cosmological scales $m_{\phi} \approx H_{0}$, while it is large in the region of high density to avoid the propagation of the fifth force $m_{\phi} \gg H_{0}$. The field that changes its mass depending on the environment is the Chameleon field. In a spherically symmetric configuration, the field equation in the Einstein frame is given by

$$
\frac{d^{2} \phi}{d r^{2}}+\frac{2}{r} \frac{d \phi}{d r}-\frac{d U_{e f f}}{d \phi}=0
$$


where $U_{\text {eff }}=U+e^{Q \phi} \rho_{m}$. A detailed discussion on thick- and thin-shell solutions in this context by finding the minima of the effective potential is available in the literature ${ }^{43-47)}$.

The thin-shell parameter is defined to be $\epsilon_{t h}=\frac{\phi_{B}-\phi_{A}}{6 Q \phi_{c}}$ and its upper bound can be found using solar system tests or the violation of the equivalence principle. If the thin-shell parameter is much less than 1, i.e., $\epsilon_{t h}<\frac{1.9 \times 10^{-5}}{Q^{2}}$, then solar system tests are satisfied even for $|Q|=O(1)$. Likewise, the propagation of the fifth force (defined by $F=-6 Q^{2} \epsilon_{t h} \frac{G M_{c}}{r}$ ) will be suppressed as compared to gravitational force if $\epsilon_{t h}<\frac{8.8 \times 10^{-7}}{Q^{2}}$, which further leads to the constraint $\left|\phi_{B}\right|<3.7 \times 10^{-15}$, where $\left|\phi_{B}\right| \gg\left|\phi_{A}\right|$. This analysis is then applied to a runaway form of the potential, e.g., the inverse power law potential, and the respective constraint on $M$ is discussed ${ }^{45,46)}$. In our case, we have found the self interacting potential with a positive power as $V=V_{c} \phi^{\frac{\beta-2}{\beta}}, \frac{\beta-2}{\beta}>0$, for negative $\beta$ values. In the present case, the minima of the effective potential inside and outside a spherically symmetric body $\left(\rho_{A} \gg \rho_{B}\right)$ are given by

$$
\phi_{A}=\left(\frac{-n V_{c}}{Q \rho_{A}}\right)^{1 / 1-n}, \quad \phi_{B}=\left(\frac{-n V_{c}}{Q \rho_{B}}\right)^{1 / 1-n} .
$$

In order to suppress the propagation of the fifth force, we must have $-n V_{c}<$ $\left(3.7 \times 10^{-15}\right)^{1-n} Q \rho_{B}$, where $\rho_{B}$ can be taken as $10^{-24} \mathrm{~g} / \mathrm{cm}^{3}$. Moreover, for the Chameleon mechanism to work, the mass of the field should be constrained by the condition $m_{A}>H_{0}$ (this allows the condition $1 /\left(m_{A} r_{c}\right) \ll 1$ ) which in our case results $\frac{(n-1)\left(-Q \rho_{A}\right)^{\frac{2-n}{1-n}}}{\left(n V_{c}\right)^{1 / 1-n}}<H_{0}$.

\section{Energy Density Perturbations}

In this section, we investigate whether the issue of structure formation is modified by the dynamics of the BD scalar field within the BI universe model. This can be achieved by considering the evolution of energy density perturbations. Basically, we perturb the field equations and take the temporal components of these equations. The behavior of the relevant variables for the accelerated expansion of the universe is investigated. For this purpose, we introduce the notations $\delta g_{\mu \nu}=h_{\mu \nu}, \delta R_{00}, \delta u^{\mu}$, and $\delta T^{00}$ to denote the perturbations in $g_{\mu \nu}, R_{00}, u^{\mu}$, and $T^{00}$, respectively. For such perturbations, we use a synchronous coordinate condition given by $h_{\mu 0}=0, \forall \mu$. Using 
perturbation in the metric tensor defined by

$$
\bar{g}_{\mu \nu}=g_{\mu \nu}+h_{\mu \nu}
$$

the perturbed form of the Ricci tensor can be written as

$$
\delta R_{\mu \nu}=\chi_{\mu \nu ; \rho}^{\rho}-\chi_{\mu \rho ; \nu}^{\rho}
$$

where

$$
\chi_{\mu \nu}^{\rho}=\frac{g^{\rho \sigma}}{2}\left[h_{\sigma \mu ; \nu}+h_{\sigma \nu ; \mu}-h_{\mu \nu ; \sigma}\right] .
$$

The non-zero temporal component of the Ricci tensor is

$$
R_{00}=-\frac{(m+2) \ddot{B}}{B}+m(1-m) \frac{\dot{B}^{2}}{B^{2}}
$$

Multiplying eq.(17) by $\frac{(m+2)}{(m+3)}$ and after some manipulation, it follows that

$$
\begin{aligned}
R_{00} & =\omega_{0} \frac{\dot{\phi}^{2}}{\phi^{2}}+\frac{\dot{\phi}}{\phi}+\frac{\rho}{\phi}\left[\frac{(m+1)\left(1-3 \gamma_{B}\right)+2(m+2)\left(2 \omega_{0}+3\right) \gamma_{B}}{(m+3)\left(2 \omega_{0}+3\right)}\right. \\
& \left.+\frac{2}{(m+3)}\right] \frac{2(1+m)}{\left(2 \omega_{0}+3\right)(m+3)}\left[\frac{V}{\phi}-\frac{V_{, \phi}}{2 \phi}\right]-\frac{(m+1) V}{(m+3) \phi} .
\end{aligned}
$$

Using the definition of the perturbed Ricci tensor given in eq.(36), the perturbed temporal component of the Ricci tensor is determined by

$$
\begin{aligned}
\delta R_{00} & =\frac{1}{2 B^{2 m}}\left[\ddot{h}_{11}-4 m \frac{\dot{B}}{B} \dot{h}_{11}+2 m\left((1+2 m) \frac{\dot{B}^{2}}{B^{2}}-\frac{\ddot{B}}{B}\right) h_{11}\right] \\
& +\frac{1}{2 B^{2}}\left[\ddot{h}_{k k}-4 \frac{\dot{B}}{B} \dot{h}_{k k}+2\left(3 \frac{\dot{B}^{2}}{B^{2}}-\frac{\ddot{B}}{B}\right) h_{k k}\right], \quad k=0,2,3 .
\end{aligned}
$$

The trace of the energy-momentum tensor is perturbed as

$$
\bar{T}=T+\delta T=T+\delta \rho-3 \delta P .
$$

The action of the perturbed D' Alembertain operator on the BD field is

$$
\begin{aligned}
\delta(\square \phi) & =\delta \ddot{\phi}+(m+2) \frac{\dot{B}}{B} \delta \dot{\phi}+\dot{\phi}\left[-\frac{1}{2}\left(\frac{\dot{h}_{11}}{B^{2 m}}\right)-\frac{1}{2}\left(\frac{\dot{h}_{k k}}{B^{2}}\right]\right. \\
& -\frac{1}{B^{2 m}} \frac{\partial^{2}}{\partial^{2} x}(\delta \phi)-\frac{1}{B^{2}} \frac{\partial^{2}}{\partial^{2} y}(\delta \phi)-\frac{1}{B^{2}} \frac{\partial^{2}}{\partial^{2} z}(\delta \phi) .
\end{aligned}
$$


We define he following parameters for relevant perturbations

$$
h_{11}=B^{2 m} h, \quad h_{k k}=B^{2} h, \quad \delta \phi=\lambda \phi, \quad \lambda \ll 1, \quad \delta \rho=\Delta \rho, \quad \Delta \ll 1 .
$$

Here, $h(t), \lambda(t)$, and $\Delta(t)$ denote the perturbed gravitational, scalar and matter density fields, respectively. Using these parameters in independent field equations, we obtain

$$
\begin{aligned}
& \ddot{h}=\ddot{\lambda}+2\left(1+\omega_{0}\right) \dot{\lambda} \frac{\dot{\phi}}{\phi}+(\Delta-\lambda) \frac{\rho}{\phi}\left[\frac{(m+1)\left(1-3 \gamma_{B}\right)}{(m+3)\left(2 \omega_{0}+3\right)}\right. \\
& \left.+\frac{2(m+2)\left(2 \omega_{0}+3\right) \gamma_{B}}{(m+3)\left(2 \omega_{0}+3\right)}+\frac{2}{(m+3)}\right]-\frac{(m+1)}{(m+3)}\left[1-\frac{\left(2-n_{1}\right)}{\left(2 \omega_{0}+3\right)}\right] V_{c}\left(n_{1}\right. \\
& -1) \lambda \phi^{n_{1}-1} \\
& \ddot{\lambda}+\dot{\lambda}\left(2 \frac{\dot{\phi}}{\phi}+(m+2) \frac{\dot{B}}{B}\right)+\lambda\left(\frac{\ddot{\phi}}{\phi}+(m+2) \frac{\dot{B}}{B} \frac{\dot{\phi}}{\phi}\right)-\frac{\dot{h} \dot{\phi}}{\phi}-\frac{1}{B^{2 m}} \frac{\partial^{2} \lambda}{\partial^{2} x} \\
& -\frac{1}{B^{2}}\left(\frac{\partial^{2} \lambda}{\partial^{2} y}+\frac{\partial^{2} \lambda}{\partial^{2} z}\right)=\frac{\Delta \rho\left(1-3 \gamma_{B}\right)}{\phi\left(2 \omega_{0}+3\right)}-\frac{\left(2-n_{1}\right) V_{c}}{\left(2 \omega_{0}+3\right)} \\
& \dot{\Delta}-\dot{h}+\delta u_{, i}^{i}=0 .
\end{aligned}
$$

Here, $u^{i}$ is the comoving velocity of the fluid and $\delta u_{, i}^{i}$ denotes the respective perturbed quantity. We also introduce the notation $n_{1}=\frac{\beta-2}{\beta}$. For the discussion of structure formation (as the structure is formed in the absence of pressure, which can prevent gravitational collapse), we consider a pressureless case, i.e., $\gamma_{B}=0$. Thus, the parameters $\alpha$ and $\beta$ turn out to be

$$
\beta=\frac{2}{1-n_{1}}, \quad \alpha=\frac{-2 n_{1}}{\left(1-n_{1}\right)(m+2)} .
$$

By using an infinitesimal gauge transformation, we can take the perturbed four-velocity as null, for which eq.(45) leads to

$$
\Delta=h,
$$

where the constant of integration is taken to be zero. We also consider a plane wave-like behavior of the considered perturbations, that is,

$$
\lambda(\mathbf{x}, t)=\lambda(t) \exp (-\iota \mathbf{k} \cdot \mathbf{x})
$$


where $\mathbf{k}$ is the wave number. Substituting eqs.(16) and (48) into eqs.(43) and (44), it follows that

$$
\begin{gathered}
\ddot{\Delta}=\ddot{\lambda}+\frac{4\left(1+\omega_{0}\right) \dot{\lambda}}{\left(1-n_{1}\right) t}+\frac{(\Delta-\lambda)}{t^{2}} \overline{\rho_{c}}\left[\frac{(m+1)+2\left(2 \omega_{0}+3\right)}{(m+3)\left(2 \omega_{0}+3\right)}\right] \\
-\frac{(m+1)}{(m+3)} V_{c}\left[1-\frac{2-n_{1}}{2 \omega_{0}+3}\right]\left(n_{1}-1\right) \lambda \frac{\phi_{0}^{\left(n_{1}-1\right)} t_{0}^{2}}{t^{2}} \\
\ddot{\lambda}=-\frac{\dot{\lambda}}{t} \frac{\left(4-2 n_{1}\right)}{\left(1-n_{1}\right)}-\frac{2 \lambda}{t^{2}\left(1-n_{1}\right)}+\frac{2 \dot{\Delta}}{t\left(1-n_{1}\right)}+\frac{\lambda k_{1}^{2}}{B_{0}^{2 m}\left(\frac{t}{t_{0}}\right)^{\frac{4 m n_{1}}{\left(1-n_{1}\right)(m+2)}}} \\
+\frac{\lambda\left(k_{2}^{2}+k_{3}^{2}\right)}{B_{0}^{2}}\left(\frac{t}{t_{0}}\right)^{\frac{4 n_{1}}{\left(1-n_{1}\right)(m+2)}}+\frac{\Delta \overline{\rho_{c}}}{\left(2 \omega_{0}+3\right) t^{2}}-\frac{\left(2-n_{1}\right) t_{0}^{2}}{\left(2 \omega_{0}+3\right) t^{2}} V_{c} \lambda n_{1} \phi_{0}^{\left(n_{1}-1\right)}
\end{gathered}
$$

where $\bar{\rho}_{c}=\frac{\rho_{c(m)}}{\phi_{0}} t_{0}^{2 /\left(1-n_{1}\right)}$. The expressions for $\rho_{c(m)}$ and $V_{c}$ in terms of $n_{1}$ are

$$
\begin{aligned}
\rho_{c(m)} & =-\frac{\phi_{0}}{t_{0}^{2 /\left(1-n_{1}\right)}}\left[\frac{m^{2}-3 m+2}{2} \frac{4 n_{1}^{2}}{\left(1-n_{1}\right)^{2}(m+2)^{2}}+\frac{2 n_{1}(m+1)}{\left(1-n_{1}\right)^{2}(m+2)}\right. \\
& \left.-\frac{2}{1-n_{1}}+\frac{(m+3) n_{1}}{\left(1-n_{1}\right)(m+2)}+\frac{4\left(1+\omega_{0}\right)}{\left(1-n_{1}\right)^{2}}\right] \\
V_{c} & =\frac{\phi_{0}^{\left(1-n_{1}\right)}}{t_{0}^{2}}\left[\frac{8 n_{1}^{2}(1+2 m)}{(m+2)^{2}\left(1-n_{1}\right)^{2}}-\frac{4 n_{1}}{\left(1-n_{1}\right)^{2}}-\frac{4 \omega_{0}}{\left(1-n_{1}\right)^{2}}\right]
\end{aligned}
$$

Combining eqs.(49) and (150) and neglecting the terms involving higher powers of $t^{-2}$, we have

$$
\ddot{\Delta}+\frac{C_{1}}{t}(\dot{\Delta}-\dot{\lambda})+\frac{C_{2}}{t^{2}}(\Delta-\lambda)+\frac{C_{3} \dot{\lambda}}{t}+\frac{c_{4} \lambda}{t^{2}}=0,
$$


where

$$
\begin{aligned}
C_{1}\left(\omega_{0}\right) & =-\frac{2}{1-n_{1}}, \\
C_{2}\left(\omega_{0}\right) & =\left[\frac{2(m+2)+2\left(2 \omega_{0}+3\right)}{(m+3)\left(2 \omega_{0}+3\right)}\right]\left[\frac{m^{2}-3 m+2}{2} \frac{4 n_{1}^{2}}{\left(1-n_{1}\right)^{2}(m+2)^{2}}\right. \\
& \left.+\frac{2 n_{1}(m+1)}{\left(1-n_{1}\right)^{2}(m+2)}-\frac{2}{1-n_{1}}+\frac{(m+3) n_{1}}{\left(1-n_{1}\right)(m+2)}+\frac{4\left(1+\omega_{0}\right)}{\left(1-n_{1}\right)^{2}}\right], \\
C_{3}\left(\omega_{0}\right) & =C_{1}\left(\omega_{0}\right)+\frac{\left(4-2 n_{1}\right)}{\left(1-n_{1}\right)}-\frac{4\left(1+\omega_{0}\right)}{\left(1-n_{1}\right)}, \\
C_{4}\left(\omega_{0}\right) & =C_{2}\left(\omega_{0}\right)+\frac{2}{1-n_{1}}+\frac{\left(m+1+2\left(2 \omega_{0}+3\right)\right)}{(m+3)\left(2 \omega_{0}+3\right)} \bar{\rho}_{c}+\left[\frac{\left(2-n_{1}\right) n_{1}}{2 \omega_{0}+3}\right. \\
& \left.+\frac{m+1}{m+3}\left(1-n_{1}\right)\left(1-\frac{2-n_{1}}{2 \omega_{0}+3}\right)\right] V_{c} \phi_{0}^{\left(n_{1}-1\right)} t_{0}^{2} .
\end{aligned}
$$

For the solution of eq.(53), we take the following assumptions:

$$
\Delta-\lambda=f(t), \quad f(t)=\xi t^{\delta}, \quad \Delta=\chi t^{\theta},
$$

where $\xi$ and $\chi$ are arbitrary constants. The substitution of the above assumption in eq.(53) leads to $\delta=\theta$ and a quadratic equation for $\theta$ given by

$$
\chi \theta^{2}+\theta\left[\chi\left(C_{3}-1\right)+\xi\left(C_{1}-C_{3}\right)\right]+C_{4}(\chi-\xi)+C_{2} \xi=0 .
$$

The roots of this equation are

$$
\begin{aligned}
\theta_{ \pm} & =\frac{1}{2 \chi}\left[\left(\chi\left(1-C_{3}\right)+\xi\left(C_{3}-C_{1}\right)\right)\right. \\
& \left. \pm \sqrt{\left(\chi\left(1-C_{3}\right)+\xi\left(C_{3}-C_{1}\right)\right)^{2}-4 \chi\left(\left(C_{2}-C_{4}\right) \xi-C_{4} \xi\right)}\right]
\end{aligned}
$$

In previous papers ${ }^{13,37)}$, the growing modes for density perturbations are calculated in the asymptotic limit of $\left|\omega_{0}\right| \gg 1$. In a similar way, the solution $\theta$ in our case turns out to be

$$
\begin{aligned}
\theta_{ \pm} & \rightarrow \frac{2 \omega_{0}}{\left(1-n_{1}\right)}\left[\left(1-\frac{\xi}{\chi}\right)\right. \\
& \pm \sqrt{\left.\left(1-\frac{\xi}{\chi}\right)^{2}+\frac{1}{(m+3)}\left[(m+1)\left(1-n_{1}\right)\left(1-\frac{\xi}{\chi}\right)-2 \frac{\xi}{\chi}\right]\right]}
\end{aligned}
$$


This shows that in the limit $\left|\omega_{0}\right| \gg 1$, the asymptotic value of $\theta$ depends on the power of the self-interacting potential $n_{1}$ and the parameter $m$ for scale factors. As $\beta$ always remains negative, $n_{1}=\frac{\beta-2}{\beta}$ will always remain positive and greater than 1 . Also, $m$ is a positive constant with $m \neq 1$ implying that $\theta_{+}$represents the growing mode for $\xi<\chi$ in both cases $0<m<1$ and $m>1$. Note that for $m=1$, the modes we obtained match with those obtained by Sen and Seshadri ${ }^{37)}$ and $n_{1}=2$ corresponds to the modes obtained by Bertolami and Martins ${ }^{13)}$. Thus, density perturbations can grow with time using the admissible negative range of $\omega_{0}$ (found in the previous section) and appropriate choices for the constants $\xi, \chi$, and $n_{1}$ as well as for $m$ in the asymptotic limits. It can be confirmed that the growing modes can also be allowed for positive BD parameter values if we take $\beta>0$ or set the arbitrary constants $\xi>\chi$. However, the first possibility remains incompatible with the accelerated expansion of the model (as it can be obtained by setting $\alpha<3 /(m+2)$ with $q>0)$.

\section{Summary and Discussion}

In this study, we have investigated the nature of the self-interacting potential and dark energy, i.e., the energy density of the BD scalar field for the BI universe model. For this purpose, we have considered the power law ansatz for the scale factors as a well as the scalar field. By taking the matter contents as perfect fluid, we find constraints for all the relevant parameters and their best-fit values. The feasible regions for these parameters are shown graphically for the two cases $m>1$ and $0<m<1$. We have also discussed the variation in the gravitational constant and the evolution of energy density perturbations for the obtained model. The results are summarized as follows.

- The obtained model is entirely based on the parameters $\alpha, \beta, \omega_{0}$, and $m$. Using the deceleration parameter, $\alpha>\frac{(m+2)}{3}$ for an accelerated expanding universe model. On the basis of $\alpha$, the bounds for $\beta$ are found which indicate that $\beta$ always remains negative for $\alpha>\frac{m+2}{3}$ and $m \neq 1, m>0$. By imposing positivity condition of energy density for both matter and scalar field, we find bounds for the BD parameter depending on these parameters. It is seen that for admissible ranges of these parameters, we have $-2 \leq \omega_{0} \leq-1.4$ and $-1.45 \leq \omega_{0} \leq-1.22$ for $m>1$ and $0<m<1$, respectively. 
- The bounds for $\beta$ provide a positive power law potential, which can be used to derive the accelerated cosmic expansion.

- Using the best-fit values for the flatness of the universe $\Omega_{m}$ and $\Omega_{\phi}$, we find the ranges for the EoS parameter $\gamma_{\phi}$. For $0<m<1$, this parameter is constrained as $-8.45<\beta<-3.225$, and for $m>1$, as $-1.8<\gamma_{\phi}<-0.23$. These ranges are compatible with the ranges suggested by the observational data ${ }^{48)}$.

- With the help of density perturbation analysis, it is shown that $\theta_{+}$ represents the growing mode for suitable choices of parameters in both cases, $0<m<1$ and $m>1$. Thus, it can be concluded that the modifications due to the dynamics of BD field do not disturb structure formation for the BI universe model.

Note that the BD constant coupling parameter has a negative range obtained by imposing the positive energy condition and is obviously inconsistent with solar system experiment bounds. It can be confirmed that, if we take negative range of $m$, e.g., $-2<m<0$, then we can have positive values of the BD parameter, which leads to the failure of the positive energy condition 13). According to recent observations, the failure of the positive energy condition is found to be more serious as the contribution of DE must be dominant as compared to matter density. This problem has widely been observed in the context of scalar tensor theories, e.g., ${ }^{14,37,49,50)}$. Recently, it has been found ${ }^{51)}$ that the accelerated expansion is incompatible with large values of the BD parameter by showing that scalar-vacuum BD equations in 5D are equivalent to the $\mathrm{BD}$ theory in $4 \mathrm{D}$ with a self-interacting potential and an effective matter field. Moreover, it has been pointed out ${ }^{52)}$ that structure formation also remains incompatible with solar system experiment bounds.

The solar system experiments constrain the BD parameter to be greater than 40,000 for a massless scalar field, but it is not true when the scalar field is massive. In this case, we define $m \gg r^{-1}$, where $r$ is the experiment scale and $m$ is the mass of potential as the spatial dynamics of scalar field can freeze for this limit. The mass scale can be taken as $m_{A U}=10^{-27}$, where the relevant scale is taken to be the Astronomical unit $\left(1 A U=10^{8} \mathrm{~km}\right)$. By comparing with this mass scale, we say that the scalar field is massive $\left(m \gg m_{A U}\right)$ or has negligible mass $\left(m \ll m_{A U}\right)$. In our case, the selfinteracting potential is $V=V_{c} \phi^{\frac{\beta-2}{\beta}}$, with $V_{c}$ given by eq.(25), $t_{0}$ is the age of the universe and $\frac{\beta-2}{\beta}>0$. If the mass of the potential term, i.e., $V_{, \phi \phi}$, is 
greater than the mass scale, then the scalar field is massive, consequently, it could be expected that all the values of the BD parameter are admissible as shown in ref. 53, where the potential term is taken to be $V=\lambda \phi^{n} ; n>0$, which is quite similar to our case when $n=\frac{\beta-2}{\beta}$ and $\lambda=V_{c}$. However, an exact estimation could be found using the solar system constraints of the Post-Newtonian parameter as shown in the literature ${ }^{54)}$. In that case, the potential is taken to be $U(\phi)=\frac{1}{2} m^{2}\left(\phi-\phi_{0}\right)^{2}$, which is a subcase of the potential derived in the present study for $\beta=-2$ and it is shown that, for $m>200 m_{A U}$, all values of $\omega>-3 / 2$ are admissible at a $2 \sigma$ confidence level.

In our recent work ${ }^{31}$, we have studied the "early- and late-time" behaviors of a BI universe model using perfect, anisotropic and magnetized anisotropic matter contents. In the current study, we impose the condition

$\alpha>\frac{3}{m+2}$ (which is necessary for a negative deceleration parameter) and to determine will be the nature of the self-interacting potential $V$, and the energy densities and pressures due to matter and the scalar field. By exploring the admissible range of the BD coupling parameter and consequently, the EoS parameters $\gamma_{B}$ and $\gamma_{\phi}$, it is concluded that these values are well-consistent with the observed values.

\section{Acknowledgement}

The authors are grateful to the Physical Society Japan for the financial support in publication.

1) S. Perlmutter, S. Gabi, G. Goldhaber, A. Goobar, D. E. Groom, I. M. Hook, A. G. Kim, M. Y. Kim, G. C. Lee, R. Pain, C. R. Pennypacker, I. A. Small, R. S. Ellis, R. G. McMahon, B. J. Boyle, P. S. Bunclark, D. Carter, M. J. Irwin, K. Glazebrook, H. J. M. Newberg, A. V. Filippenko, T. Matheson, M. Dopita and W. C. Couch: Astrophys. J. 483 (1997) 565; S. Perlmutter, G. Aldering, M. D. Valle, S. Deustua, R. S. Ellis, S. Fabbro, A. Fruchter, G. Goldhaber, A. Goobar, D. E. Groom, I. M. Hook, A. G. Kim, M. Y. Kim, R. A. Knop, C. Lidman, R. G. McMahon, P. Nugent, R. Pain, N. Panagia, C. R. Pennypacker, P. Ruiz-Lapuente, B. Schaefer and N. Walton: Nature 391 (1998) 51; S. Perlmutter, G. Aldering, G. Goldhaber, R. A. Knop, P. Nugent, P. G. Castro, S. Deustua, S. Fabbro, A. Goobar, D. E. Groom, I. M. Hook, A. G. Kim, M. Y. Kim, J. C. Lee, N. J. Nunes, R. Pain, C. R. Pennypacker, R. Quimbey, C. Lidman, R. S. Ellis, M. Irwin, R. G. Mcmahon, P. Ruiz-lapuente, N. Walton, B. Schaefer, B. J. Boyle, A. V. 
Filippenko, T. Matheson, A. S. Fruchter, N. Panagia, H. J. M. Newberg, and W. J. Couch: Astrophys. J. 517 (1999) 565.

2) A. G. Riess, A. V. Filippenko, P. Challis, A. ClocChiatti, A. Diercks, P. M. Garnavich, R. L. Gilliland, C. J. Hogan, S. Jha, R. P. Kirshner, B. Leibundgut, M. M. Phillips, D. Reiss, B. P. Schmidt, R. A. Schommer, R. C. Smith, J. Spyromilio, C. Stubbs, N. B. Suntzeff, and J. Tonry: Astron. J. 116 (1998) 1009.

3) C. L. Bennett, M. Halpern, G. Hinshaw, N. Jarosik, A. Kogut, M. Limon, S. S. Meyer, L. Page, D. N. Spergel, G. S. Tucker, E. Wollack, E. L. Wright, C. Barnes, M. R. Greason, R. S. Hill, E. Komatsu, M. R. Nolta, N. Odegard, H. V. Peiris, L. Verde, and J. L. Weiland: Astrophys. J. Suppl. 148 (2003) 1.

4) M. Tegmark, M. A. Strauss, M. R. Blanton, K. Abazajian, S. Dodelson, H. Sandvik, X. Wang, D. H. Weinberg, I. Zehavi, N. A. Bahcall, F. Hoyle, D. Schlegel, R. Scoccimarro, M. S. Vogeley, A. Berlind, T. Budavari, A. Connolly, D. J. Eisenstein, D. Finkbeiner, J. A. Frieman, J. E. Gunn, L. Hui, B. Jain, D. Johnston, S. Kent, H. Lin, R. Nakajima, R. C. Nichol, J. P. Ostriker, A. Pope, R. Scranton, U. Seljak, R. K. Sheth, A. Stebbins, A. S. Szalay, I. Szapudi, Y. Xu, J. Annis, J. Brinkmann, S. Burles, F. J. Castander, I. Csabai, J. Loveday, M. Doi, M. Fukugita, B. Gillespie, G. Hennessy, D. W. Hogg, Z. E. Ivezic, G. R. Knapp, D. Q. Lamb, B. C. Lee, R. H. Lupton, T. A. McKay, P. Kunszt, J. A. Munn, L. Connell, J. Peoples, J. R. Pier, M. Richmond, C. Rockosi, D. P. Schneider, C. Stoughton, D. L. Tucker, D. E. V. Berk, B. Yanny, and D. G. York: Phys. Rev. D 69 (2004) 03501.

5) S. W. Allen, R. W. Schmidt, H. Ebeling, A. C. Fabian, and L. V. Speybroeck: Mon. Not. Roy. Astron. Soc. 353 (2004) 457.

6) E. Hawkins, S. Maddox, S. Cole, O. Lahav, D. S. Madgwick, P. Norberg, J. A. Peacock, I. K. Baldry, C. M. Baugh, J. Bland-Hawthorn, T. Bridges, R. Cannon, M. Colless, C. Collins, W. Couch, G. Dalton, R. D. Propris, S. P. Driver, S.P., G. Efstathiou, R. S. Ellis, C.S. Frenk, K. Glazebrook, C. Jackson, B. Jones, I. Lewis, S. Lumsden, W. Percival, B. A. Peterson, W. Sutherland, and K. Taylor: Mon. Not. Roy. Astr. Soc. 346 (2003) 78.

7) B. Jain and A. Taylor: Phys. Rev. Lett. 91 (2003) 141302.

8) A. S. Al-Rawaf and M. O. Taha: Gen. Relativ. Gravit. 28 (1996) 935.

9) R. R. Caldwell, R. Dave, and P. J. Steinhardt: Phys. Rev. Lett. 80 (1998) 1582.

10) P. J. Steinhardt, L. Wang, and I. Zlatev: Phys. Rev. Lett. 59 (1999) 123504 . 
11) I. Zlatev, L. Wang, and P. J. Steinhardt: Phys. Rev. Lett. 82 (1999) 896.

12) L. A. U. Lopez and T. Matos: Phys. Rev. D 62 (2000) 081302.

13) O. Bertolami and P. J. Martins: Phys. Rev. D 61 (2000) 064007.

14) N. Benerjee and D. Pavon: Phys. Rev. D 63 (2001) 043504.

15) B. K. Sahoo and L. P. Singh: Mod. Phys. Lett. A 18 (2003) 2725.

16) W. Chakraborty and U. Debnath: Int. J. Theor. Phys. 48(2009)232.

17) C. H. Brans and R. H. Dicke: Phys. Rev. 124(1961)925.

18) S. Weinberg: Gravitation and Cosmology (Wiley, 1972).

19) P. A. M. Dirac: Proc. R. Soc. Lond. A 165 (1938) 199.

20) B. Bertotti, L. Iess, and P. Tortora: Nature 425 (2003) 374.

21) A. D. Felice, G. Mangano, P. D. Serpico, and M. Trodden: Phys. Rev. D 74 (2006) 103005.

22) S. K. Rama and S. Gosh: Phys. Lett. B 383 (1996) 32; S. K. Rama: Phys. Lett. B 373 (1996) 282.

23) C. Romero and A. Barros: Phys. Lett. A 173 (1993) 243.

24) N. Benerjee and S. Sen: Phys. Rev. D 56 (1997) 1334.

25) J. P. Singh and P. S. Baghel: Elect. J. Theor. Phys. 6 (2009) 85.

26) M. K. Verma, M. Zeyauddin, and S. Ram: Rom. J. Phys. 56 (2011) 616.

27) H. K. Eriksen, F. K. Hansen, A. J. Banday, K. M. Gorski, and P. B. Lilje: Astrophys. J. 605 (2004) 14.

28) T. R. Jaffe, A. J. Banday, H. K. Eriksen, K. M. Gorski, and F. K. Hansen: Astrophys. J. 629 (2005) L1.

29) E. G. King and P. Coles: Class. Quantum Grav. 24 (2007) 2061.

30) T. Singh and L. N. Rai: Gen. Relativ. Gravit. 15 (1983) 875.

31) M. Sharif and S. Waheed: Eur. Phys. J. C 72 (2012) 1876.

32) M. S. Bermann: Nuovo Cimento B 74 (1983) 192.

33) J. Khoury and A. Weltman: Phys. Rev. D 69 (2004) 044026.

34) R. de Ritis, A. A. Marino, C. Rubano, and P. Scudellaro: Phys. Rev. D

62 (2000) 043506.

35) S. Sen and A. A. Sen: Phys. Rev. D 63 (2001) 124006.

36) V. Faraoni: Phys. Rev. D 62 (2000) 023504.

37) S. Sen and T. R. Seshadri: Int. J. Mod. Phys. D 12 (2003) 445.

38) M. Sharif and M. Zubair: Astrophys. Space Sci. 330 (2010) 399.

39) C. B. Collins, E. N. Glass, and D. A. Wilkinson: Gen. Relativ. Gravit. 12 (1980) 805.

40) K. S. Throne: Astrophys. J. 148 (1967) 51.

41) J. Kristian and R. K. Sachs: Astrophys. J. 143 (1966) 379. 
42) C. B. Collins: Phys. Lett. A 60 (1977) 397.

43) J. Khoury and A. Weltman: Phys. Rev. Lett. 93 (2004) 171104.

44) J. Khoury and A. Weltman: Phys. Rev. D 69 (2004) 044026.

45) S. Tsujikawa, K. Uddin, S. Mizuno, R. Tavakol, and J. Yokoyama: Phys. Rev. D 77 (2008) 103009.

46) S. Tsujikawa, T. Tamaki, and R. Tavakol: JCAP 0905 (2009) 020.

47) D. F. Mota and H. A. Winther: Astrophys. J. 733 (2011) 7.

48) R. R. Caldwell: Phys. Lett. B 545 (2002) 23.

49) A. A. Sen, S. Sen, and S. Sethi: Phys. Rev. D 63 (2001) 107501.

50) A. F. Bahrehbakhsh, M. Farhoudi, and H. Shojaie: Gen. Relativ. Grav.

43 (2011) 847.

51) J. P. D. Leon: Class. Quantum Grav. 27 (2010) 095002.

52) E. Gaztanaga and J. A. Lobo: Astrophys. J. 548 (2001) 47.

53) L. Perivolaropoulos: Phys. Rev. D 67 (2003) 123516.

54) L. Perivolaropoulos: Phys. Rev. D 81 (2010) 047501. 